\title{
On-demand Multipath Routing Protocols for Mobile Ad-Hoc Networks: A Comparative Survey
}

\author{
Tariq A. Murshedi, Xingwei Wang, and Hui Cheng
}

\begin{abstract}
A Mobile Ad Hoc Network (MANET) is an infrastructure-less, self-organized and multi-hop network with a rapidly changing topology causing the wireless links to be broken at any time. Routing in such a network is challenging due to the mobility of its nodes and the challenge becomes more difficult when the network size increases. Due to the limited capacity of a multi-hop path and the high dynamics of wireless links, the single-path routing approach is unable to provide efficient high data rate transmission in MANETs. The multipath routing is the routing technique of using multiple alternative paths through a network. Furthermore, whenever a link failure is detected on a primary route, the source node can select the optimal route among multiple available routes. Therefore, the multipath routing approach is broadly utilized as one of the possible solutions to overcome the single-path limitation. Most of the multipath routing protocols are based on Ad Hoc On-Demand Distance Vector (AODV). The objective of this paper is to provide a survey and compare sets of multipath routing protocols for mobile ad-hoc networks. This survey will motivate the design of new multipath routing protocols, which overcome the weaknesses identified in this paper.
\end{abstract}

Index Terms-Mobile ad-hoc networks, on-demand multipath routing, single path routing, dynamic source routing, ad hoc on-demand distance vector.

\section{INTRODUCTION}

In the last decades, mobile ad hoc networks (MANETs) [1], [2] have attracted amounts of attention. A mobile ad hoc network consists of a collection of wireless mobile nodes (or routers) dynamically forming a temporary network without using any existing infrastructure or centralized administration. The routers in MANETs are free to move and organize themselves; thus, the network topology changes rapidly and unpredictably. MANETs are characterized by multi-hop, mobility, large device heterogeneity, limited bandwidth, and limited battery energy supply. Due to these characteristics, a routing path connecting the source node to the destination node may be broken at any time, bringing a major challenge to the design of routing protocols in MANETs.

Routing [3], [4] is the process of searching and maintaining routes between the source node and the destination node in the network. A routing protocol is needed whenever a packet needs to be transmitted via a number of

Manuscript received March 12, 2016; revised May 9, 2016.

Tariq A. Murshedi and Xingwei Wang are with the College of Information Science and Engineering, Northeastern University, Shenyang 110819, China (e-mail: tariq_alwan@yahoo.com, wangxw@mail.neu.edu.cn).

Hui Cheng was with School of Computing and Mathematical Sciences, Liverpool John Moores University, Liverpool L3 3AF, UK (e-mail: H.Cheng@ljmu.ac.uk). intermediate nodes. For MANETs, there are mainly two kinds of routing protocols, i.e., table-based protocols (called proactive protocols) and on-demand protocols (called reactive protocols). In table-based protocols, each node maintains a routing table, which contains routes to all the other nodes in the network. Nodes must periodically exchange routing information to keep routing tables up-to-date. Therefore, routes between nodes are computed and stored, even when they are not needed. Table-based protocols will be impractical for large and highly dynamic networks.

Unlike table-based protocols, on-demand routing is a popular routing category for mobile ad hoc networks. It is arelatively new routing philosophy that provides a scalable solution to large size of network topologies. The design follows the idea that each node sends routing packets only when communication is requested. By this way, the routing overhead is reduced. An on-demand routing protocols consists of the following two main phases. First, route discovery is conducted to search a route between two nodes. Second, route maintenance is conducted to repair a broken route or search a new route in the presence of route failures. There are different types of on-demand routing protocols such as Dynamic Source Routing (DSR) [5] and Ad Hoc On-Demand Distance Vector (AODV) [6]

In MANETs [7], [8], another classification of routing protocols can be made in terms of the number of paths one protocol delivers per source destination pair. There exist unipath and multipath routing protocols. For a unipath routing protocol, a single route is used to deliver data from the source node to the destination node. Most of the routing protocols in MANETs form a single-path, such as DSR and AODV. Single-path routing protocols need to repair routes each time the route is broken. This route repair generates many control packets, and increases the end-to-end delay. In order to overcome these drawbacks of the single path routing, multipath routing schemes have been proposed. In a multipath routing protocol, more than one route is used to deliver the data. Multipath routing [9] is a technique, which can improve the reliability of the transmission. The objective of designing a multipath routing protocol is to provide enhanced robustness to node or link failures. If one could provide multiple paths from a source to a destination, one could envision that the transmission of redundant information on various paths would help the receiver reconstruct the transmitted information even if a few of the paths were to fail. In addition, multipath routing has the advantages of balancing load, minimizing end- to-end delay, increasing fault-tolerance, reducing the frequency of route inquiries and achieving a lower overall routing overhead.

The rest of this paper is organized as follows. Section II 
summarizes the challenges in designing multipath routing protocols. Section III briefly explains on-demand routing protocols for MANETs. Section IV provides detailed operational descriptions, objectives, limitations and comparison of the multipath protocols. Section V concludes the paper and discusses possible lines of future work.

\section{Challenges In Designing Multipath Routing PROTOCOLS}

While designing a multipath routing protocol, the following three major challenges have been addressed in the literature [10]-[12].

\section{A. How to Discover Multiple Paths}

The basic route discovery process of on-demand routing protocols like AODV is usually used to discover multiple routes from the source to the destination. This route discovery mechanism needs to be modified in order for it to discover multiple paths. In many multipath routing applications, disjoint paths are favored due to the independence of the paths. There are two types of disjoint paths, i.e., node-disjoint and link-disjoint. Node-disjoint paths do not have any nodes in common, except the source and the destination. In contrast, link-disjoint paths do not have any link in common. Hence, the route discovery mechanisms of the existing routing protocols need to be modified to discover a maximum number of node-disjoint or link-disjoint paths.

\section{B. How to Select a Path}

A multipath routing protocol should decide how to select a path for sending data packets after all the routes are discovered. According to Lee et al. [13] if a number of routes are discovered, it is required to know how many of these routes should be used (some or all of them). If only a small number of paths are used, the performance of a multipath routing protocol would be similar as that of the shortest path routing protocol. On the other hand, if all these paths are used, there is a chance of selecting an excessively long path, which may adversely affect the performance of a multipath routing protocol.

\section{How to Distribute the Load}

A multipath routing protocol should determine how to use these multiple routes during the transmission of data packets. When a path or a set of paths are selected, there arises another challenge, specifically, how a source node should send a packet. It may divide a packet stream into several parts and send these segments by using different paths or it may send duplicate copies of a packet by using different paths.

\section{ON-DEMAND ROUTING PROTOCOLS FOR MANETS}

Routing in mobile ad hoc networks presents a great challenge, which becomes more difficult when the network size becomes larger. The challenge comes mainly from two aspects: 1) node mobility, which causes frequent topological changes, and 2) limited network bandwidth, which restricts the timely topological updates at each router [14], [15]. For On-demand protocols, a route between nodes is initiated whenever there is a desire to establish a link. This is done via a routing discovery process, which is initiated whenever there is a need to transmit data packets to a destination [16]. Most multipath routing protocols mentioned in this paper are based on the principle of the AODV protocol as shown in Fig. 1. However, all multipath routing protocols share a common characteristic, i.e., they discover multiple routes between a pair of source-destination nodes [17].

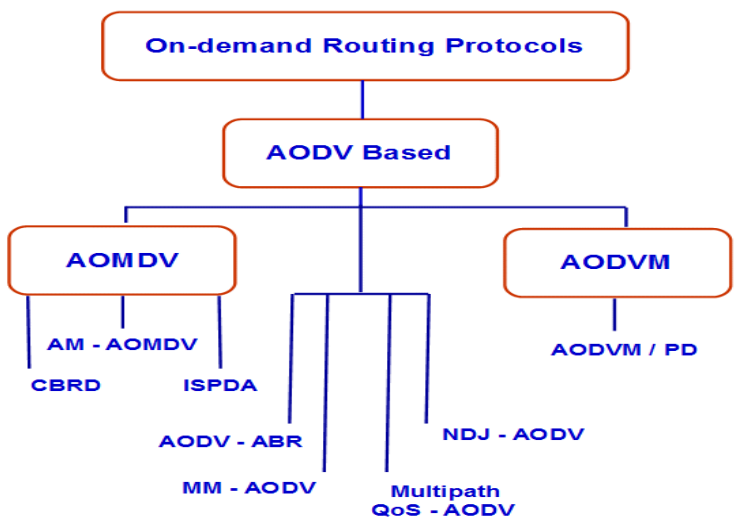

Fig. 1. Multipath routing protocols.

\section{Multipath Routing Protocols}

Multipath routing protocols generally are considered more reliable and robust than single-path routing protocols [18]. Furthermore, whenever a link failure is detected on a primary route, the source node can select the optimal route among the other available routes. This mechanism enhances route availability and consequently reduces control overhead. It also saves energy, enhances data transmission rate, and increases the network bandwidth. Recently, several multipath routing protocols have been proposed, and many of them are based on AODV. In this section, we will present a selection of them.

\section{A. On-demand Multipath Distance Vector Routing Protocol}

A multipath extension of AODV protocol called on demand multipath distance vector (AOMDV) protocol was proposed by Marina and Das [19]-[21]. The main idea of AOMDV protocol is to provide efficient fault tolerance in the sense of fast and efficient recovery from route failures in highly dynamic networks where link failures and route breakages occur frequently. It reduces end-to-end delay more than a factor of two, and is also able to reduce routing overhead and the frequency of route discoveries by about $20 \%$ but increases the number of delivered packets. The AOMDV protocol consists of two main components, i.e., a rule for route updates to find multiple loop-free paths at each node, and a distributed protocol to compute the link-disjoint paths.

In this protocol, each route arriving at a node during route discovery potentially defines an alternative route to the source or the destination. Accepting all of them to construct routes will lead to routing loops. In order to eliminate any possible loops, the AOMDV uses new metric of advertised hop count. The advertised hop count represents the maximum hop counts of each of those available paths. The protocol only allows accepting alternative route with lower hop counts. This metric is necessary to guarantee loop free. The AOMDV protocol computes link-disjoint paths per route discovery. 
With multiple redundant paths available, the protocol switches routes to a different path when an earlier path fails. Thus, a new route discovery is avoided. For efficiency, only link disjoint paths are computed so that the paths fail independently of each other.

Note that link-disjoint paths are sufficient in AOMDV that is, using multipath routing for reducing routing overhead rather than for load balancing. Therefore, node-disjoint paths are more useful, as switching to an alternative route is guaranteed to avoid any congested node. The AOMDV protocol [22], [23] achieves the best performance in high mobility scenarios. When increasing the network density, the protocol performance decreases and it has the additional overhead of more RREPs per route discovery.

\section{B. On-demand Distance Vector Multipath}

Ye et al. [9], [18] proposed a multipath extension of AODV protocol called on demand distance vector multipath (AODVM) protocol. The main goal of AODVM is to primarily design a multipath routing framework for providing enhanced robustness to node failures. In order to provide the reliability of paths and security, AODVM introduces reliable path segments, which are formed by reliable nodes. Nodes that join two segments have to be reliable nodes.

In AODVM protocol, instead of discarding the duplicate RREQ packets, intermediate nodes store the information contained in these packets in a table called RREQ table. When an intermediate node receives a RREQ packet, it records the following information in its RREQ table: the source ID, the destination ID, the neighbor list as shown in Fig. 2. Furthermore, an intermediate node located between a source and a destination is not allowed to send a reply to the source.

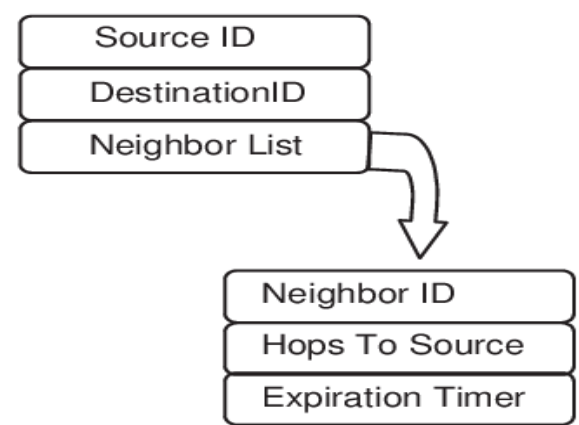

Fig. 2. The Structure of each RREQ table entry in AODVM.

When the destination receives the RREQ packet, it updates its sequence number and generates a RREP packet. The RREP packet contains an additional field called last hop ID to indicate the neighbor from which the particular copy of RREQ packet is received. A destination node generates RREP packets for each RREQ packet received from its neighbors. When an intermediate node receives a RREP packet from a neighbor, it deletes the entry corresponding to this neighbor from its RREQ table and adds a routing entry to its routing table (shown in Fig. 3) to indicate the discovered route from itself to a destination node. The node determines the neighbor in the RREQ table via the shortest path to the source, and forwards the RREP packet to that neighbor. Then, the entry corresponding to this neighbor is deleted from the RREQ table. When an intermediate node receives RREP packet and if it is unable to forward received packet (no entries in its RREQ table), it generates a Route Discovery Error (RDER) packet and sends it to the neighbor that the $\mathrm{RREP}$ is received from.

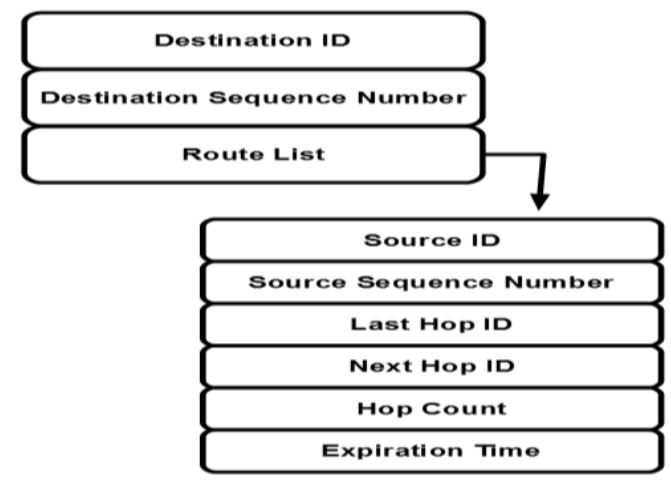

Fig. 3. Structure of the each routingtable entry in AODVM.

The neighbor, upon receiving the RDER packet, will try to forward the RREP to a different neighbor, which forwards it further towards the source. Since an intermediate node makes decisions on where to forward the RREP packet (unlike in source routing), a source node and a destination node are unaware of that forwarding decision. Thus, when a source node receives a RREP packet, it should confirm each received RREP packet by sending a route request confirmation message (RRCM).

In AODVM protocol, the sequence number is used to prevent loops. When a source node initiates a RREQ, it increases its sequence number and the destination sequence number. If the destination receives a new RREQ packet, it computes a new sequence number and includes it in the RREP packet.

\section{Multipath AODV with Path Diversity Protocol}

Mueller and Ghosal [24] proposed a multipath extension of AODVM protocol called on demand distance vector multipath with path diversity (AODVM/PD) protocol. The main objective of AODVM/PD protocol is to find diverse paths with a correlation factor between paths. It provides both smaller end-to-end delay than AODVM in networks with low mobility and better fault tolerance.

In AODVM/PD, the route discovery process is similar to that of the AODVM protocol. The main difference between AODVM/PD and AODVM is that AODVM/PD depends upon the correlation factor metric. The correlation factor of two node-disjoint paths is defined as the total number of links connecting the paths. In order to implement AODVM/PD, a node maintains three parameters during route discovery: 1) Local Correlation Factor ( $L C F$ ), which measure of how many RREPs associated with a given route discovery that a node has overheard. 2) Area Correlation Factor (ACF), which is the weighted average of a node's local correlation factor and the average of its neighbors' local correlation factors and 3) Correlation Threshold (CT), when a node's area correlation factor is over the correlation threshold, the node is no longer allowed to participate in any routes for the particular route discovery.

In AODVM/PD protocol, a modification is made to the route reply and route confirmation phases of AODVM. When a node overhears a RREP packet, it increments its local correlation factor by one as shown in Fig. 4. When a node receives a RREP addressed to it and its $A C F$ is greaterthan the 
$C T$, it sends a Route Discovery Error (RDER) packet to the sender. Otherwise, the node selects the neighbor from the RREQ table with the shortest hop count to the source, and forwards the RREP to that neighbor. If a node overhears an RRCM, it broadcasts a Correlation (CORR) packet containing its local correlation factor. When a node receives a CORR packet from a neighbor, it updates the local correlation of its neighbor in the RREQ table. The node then calculates its $A C F$. If its $A C F$ is greater than the $C T$, then it broadcasts another CORR packet with the OVER_THRESHOLD flag set to true. When a node receives a CORR packet with the OVER_THRESHOLD flag set, it deletes the sending node from its RREQ table. When the destination receives an RRCM, it sends the next RREP to a neighbor from its RREQ table. The purpose of the route confirmation phase is to remove nodes with a relatively high area correlation from RREQ tables.

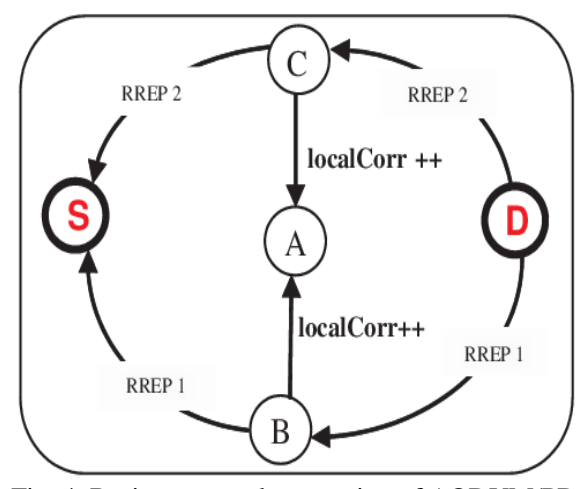

Fig. 4. Basic route reply operation of AODVM/PD.

The AODVM/PD protocol has two drawbacks as follows. First, when the mobility level of the nodes increases, the delay becomes greater than AODVM. Second, the control overhead is increased due to the transmission of CORR packet during the route discovery phase.

\section{Adaptive Backup Routing Protocol}

AODV-BR (AODV with Backup Routes) is an AODV-based protocol. It creates a mesh and provides multiple alternate routes for each desired destination [18]. A multipath extension of AODV-BR protocol called adaptive backup routing for mobile ad-hoc networks (AODV-ABR) protocol was proposed by Lai et al. [19], [25]. It aims to reduce control overhead. The mesh structure can be created by overhearing the data packets transmitted from neighbor nodes. This helps to increase the adaptation to topology changes without transmitting extra control messages.

In AODV-ABR protocol, the operations are similar to the original AODV. Therefore, each routing table and alternate route table also contain the following information: Destination IP, next hop, hop count, destination sequence number, and expiration time. When a node detects a broken link, it will perform a handshake process with its immediate neighbors to repair the broken route instead of an one-hop data broadcast to its immediate neighbors. The handshake procedure is accomplished by two one-hop control signals: BRRQ (Backup Route Request) and BRRP (Backup Route Reply). Fig. 5 is an example showing how this process is accomplished. When the link between node $\mathrm{B}$ and node $\mathrm{C}$ breaks, node B will broadcast a BRRQ signal to its neighbors. Then node $\mathrm{E}$ and node $\mathrm{F}$ will send BRRP with the hop count to node $\mathrm{B}$. Node $\mathrm{B}$ will choose node $\mathrm{F}$ as the next hop to the destination, and then transmit data packets to node F. Node B and $F$ will update its routing tables to reflect these changes.

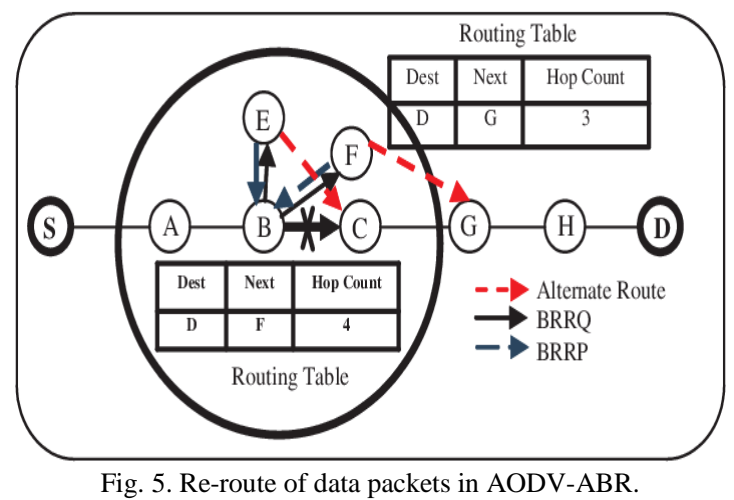

In this protocol, AODV-LR (Local Repair) repairs the link locally if the broken link is not far away from the destination, but AODV-ABR could repair the link anywhere along the primary route if alternate routes exist. Therefore, AODV-ABL (Adaptive Backup Route and Local repair) process combines AODV-ABR with AODV-LR algorithm. If the distance between the broken link and the destination is less than MAX REPAIR TTL hops, AODVABL would try to repair the link by broadcasting a RREQ control signal as AODV-LR, otherwise the AODV-ABL will repair the link by a handshake process with immediate neighbors. When the data transmission rate is exceeded to 8 packets per second, AODV-ABL still has the best performance and AODV-ABR becomes slightly better than AODV-LR. The traffic load will become heavier, when more nodes participate in a wireless network. The probability of packet collisions will increase, resulting in the degradation in overall performance.

\section{E. Adaptive Multi-metric On-demand Multipath Distance Vector Routing}

Khimsara et al. [26] proposed adaptive multi-metric on-demand multipath distance vector routing (AM-AOMDV) protocol as an extension to the AOMDV protocol, which considers multi-metric and local route update as the most important factors in selecting routes. The main objective of AM-AOMDV is to increase the packet throughput and route longevity, and decrease the end-to-end latency, route discovery frequency and route overhead in high mobility environments.

There are three metrics included in AM-AOMDV: node-to-end Received Signal Strength Indication (RSSI), node-to-end latency and node occupancy, in addition to the minimum hop-count to select the most efficient route from the source to the destination. The multiple metrics also help in avoiding the hot spots under heavy traffic conditions. These metric values are recorded in the routing table for each node and their corresponding paths. The node-to-end RSSI metric is defined as the RSSI value of the path from any node to the destination. It uses the average value of RSSI. The RSSI value of each forward link is fedbackto the nodes through the ACK packets. The node-to-end latency consists of two parameters: 1) delay from the node to the source computed from the timestamp of the RREQ packet, and 2) the delay from the destination to the node computed from the RREP packet. The node occupancy is defined as the total 
number of data packets that any node processes per second and it plays an important role under heavy traffic conditions.

In AM-AOMDV, the route discovery procedure is similar to the AOMDV protocol. The source node begins forwarding packets, after receiving the first RREP from the destination. If a new RREP arrives, the source will change to the new route. This is because the protocol is adaptive and chooses the best available route, unlike AOMDV. The AM-AOMDV updates the route to the destination periodically using the local update algorithm. The local path updates in AM-AOMDV increase the route longevity. The end-to-end latency, the route discovery latency and routing overhead are much lower than in the AOMDV and hence it is more reliable than AOMDV. In a sparse network with lower number of connections, the AM-AOMDV shows higher route discovery delay than AOMDV.

\section{F. Congestion Based Route Discovery Protocol}

Bawa and Banerjee [27] proposed a congestion based route discovery (CBRD) protocol as an extension to AOMDV protocol. The main advantage of this protocol is to check congestion on a node and then apply load balancing. The CBRD also aims at improving the throughput, delay and reducing packet loss during transmission as compared to AOMDV protocol.

The route maintenance of CBRD is similar to that of the AOMDV protocol, but route discovery is modified to find a link-disjoint as well as a node-disjoint path from the source to the destination. The main difference between CBRD and AOMDV is that CBRD considers the queue size of node while making a routing decision. When a source node sends a packet to a destination, the packet includes some value for congestion. When intermediate nodes receive the RREQ packet, they check the value against their queue. If the queue size is sufficient then that node can participate in the communication. Otherwise, the node discards it. The source node calculates congestion at each node and selects the best route. Packets are transferred based on minimum congestion on the route. At the end the best path is selected for communication and set as the primary path. Other paths are set as secondary paths for backup, which are used when the primary path breaks up. Priorities are also set on paths to choose next primary path. When the route is congested, the source chooses other paths for data transmission.

The CBRD protocol has two drawbacks as follows. First, when a node moves to other side trace file, the delay begins to increase. Second, some packets are lost when a node moves in another direction.

\section{G. Improved Stability Based Partially Disjoint AOMDV}

Improved stability based partially disjoint (ISPDA) protocol was developed by Almobaideen et al. [28] as an improvement on the stability based partially disjoint AODVM (SPDA) protocol which considers node stability and hop count values as the most important factors in selecting a route. The main objective of ISPDA protocol is to improve the performance of SPDA protocol by overcoming two weaknesses. First, SPDA does not take into consideration the number of hops of each path. Second, SPDA transmits packets over the shortest path until it becomes invalid before it tries to utilize other alternative paths.

Disjoint multipath routing protocols have been classified into maximally disjoint multipath (e.g. AOMDV protocol) and partially disjoint multipath protocols (e.g. ISPDA). The shared nodes or links between the partially disjoint paths are selected based on stability. Stability of the routes in MANET can be measured based on node stability or link stability. The ISPDA protocol chooses the most stable paths with the minimum number of hops. This increases the lifetime of the selected paths and so reduces the opportunity of future path breaks, which in turn reduces packet transmission delay. This protocol transmits packets in parallel over all the discovered paths. It starts the transmission with the first discovered path.

In ISPDA, the destination receives the first RREQ packet via a route that will be considered as a primary route and through which a RREP packet is sent back to the source node. When the destination receives other RREQs, it will firstly check the number of hop counts. If this new path is shorter or equal to the primary route, then the destination checks the stability of each node on this path and it chooses the shortest paths with more stable links. The selected alternative paths will be used in parallel to transmit the data by the sender node in order to increase the utilization of available bandwidth.

In this protocol, the node stability is estimated based on the number of RREQs that passed through the node contained in the list of nodes carried by the receiving RREQs. An intermediate node is considered stable based on two factors. First, the number of times that a node has been traversed on various paths is greater than specific threshold. Second, the time of the last occurrence, when that node has been encountered on a path, is less than a specific period.

ISPDA protocol outperforms SPDA protocol in terms of average end-to-end delay and throughput, but the discovery overhead of ISPDA is higher than that of SPDA.

\section{H. A Novel Approach to Find the Complete Node-Disjoint Multipath in AODV}

A multipath extension of AODV protocol called maximum multipath AODV (MM-AODV) was proposed by Chowdhury and Mukta [29]. The main motivation of this protocol is to discover maximum available complete node-disjoint paths between a pair of nodes depending on the number of source and destination neighbors nodes. The MM-AODV protocol balances energy and traffic load of overall network to increase the network lifetime. This approach uses the same principle of AODV protocol, but the memory storage is increased, to storethe alternative paths.

In MM-AODV protocol, the source node initiates route discovery procedure by broadcasting the RREQ message. When neighbor nodes of the source receive RREQ, the nodes so called secondary sources, include their addresses in the message. RREQ packet contains the source address, source sequence number, broadcast id, destination address, destination sequence number, secondary source address, andhop count. If secondary source is different, neighbor nodes of destination allow two duplicate messages. The destination node generates RREP for each secondary source. For secondary sources with multiple route replies, the first one will be used and the second one will be stored.

In this protocol, when the first link fails, the intermediate node uses an RERR message to notify other nodes that the 
loss of that link has occurred. When the source node gets an RERR message, it can check if any valid route is available through the same intermediate node using the following conditions.

Each path from the neighbors of destination:

1) If two secondary source paths share a path, only one path is selected.

2) When two paths overlap with the same secondary source, the first one will be selected and the second one will be stored.

3) If neighbor nodes of destination are fewer than secondary sources, a number of selected paths are not more than a number of destination neighbors.

4) If all available paths are fewer than neighbors of source and/or destination due to intermediate nodes, a number of selected paths can be less than source neighbors or destination neighbors.

Each route reply builds a single path to each secondary source and source node can build multipath with it. The MM-AODV protocol builds maximum complete node-disjoint multipath. After the discovery of multipath, they can be used for several ways such as security, traffic load, and energy distribution.

The MM-AODV protocol consists of two drawbacks as follows. First, when node speed increases, path break probability also increases, therefore the packet delivery rate of MM-AODV becomes slightly less than AODV. Second, the memory storage is increased, as the alternative paths are stored.

\section{NDj-AODV: Node Disjoint Multipath Routing inMobile Ad Hoc Networks Based on AODV Protocol}

Arya and Gandhi [30] proposed node disjoint multipath routing in mobile ad hoc networks (NDj-AODV) based on AODV Protocol. The principal objective of NDj-AODV is to find multiple node-disjoint paths to a destination with less routing overhead and low route discovery latencies. Node-disjoint paths do not have any node in common and are usually less in number as compared to link-disjoint paths. $\mathrm{NDj}$-AODV uses the concept of overhearing by intermediate nodes and is able to find a maximum number of node-disjoint paths towards a destination.

When a source node does not have a route to the destination, it floods an RREQ packet into the network. If a node receives an RREQ packet, it caches the source address, broadcast id, previous hop address and hop count. Caching previous hop address and hop count allow intermediate nodes to process multiple RREQs. When a destination node receives an RREQ, it firstly increments a counter maintained for RREQs. The first RREP is sent immediately for the first RREQ received from the intermediate node. For subsequent RREQ's delayed RREP's are sent which can be done using exponential delays. This aid in discovering node disjoints paths.

The NDj-AODV protocol allows intermediate nodes to overhear RREP packets forwarded by neighboring nodes to upstream nodes. On receiving an RREP packet, an overhearing node takes a decision based on Algorithm 1. When a node forwards an RREP to a node for a reverse route entry stored in its routing table, a neighboring node overhears this transmission. The neighboring node checks whether it has a reverse route to the destination (previous hop that forwarded a RREQ) of this RREP stored in its routing table. If the reverse route exists, it deletes the route from its routing table. This helps in finding node-disjoint paths as no intermediate node will store routes to previous hop nodes to which an RREP has been already sent previously by another node.

Algorithm 1: NDj-AODV Route Discovery Process when a node forwards a RREP packet.

$\mathrm{S}=$ Address of source node

$\mathrm{N} \chi=$ Node that forwards RREP packet

$\mathrm{N} \gamma=$ Node that overhears transmission of RREP packet by $\mathrm{N} \chi$

PrevHop_Node $=$ Address of next hop to which

RREP is sent

Allow promiscuous / overhearing mode at intermediate nodes

N $\chi$ forwards REEP packet to PrevHop_Node for

which a reverse route is stored in its routing table.

$\mathrm{N} \gamma$ overhears this transmission

At node $\mathrm{N} \gamma$

If (route to PrevHop_Node exists \&\&HopCount

to Source $>1$

thendelete_route_entry (PrevHop_Node)// Delete

route stored for PrevHop_Node from the

routing table of node $\mathrm{N} \gamma$

endif

The NDj-AODV protocol has three drawbacks as follows. First, it achieves a slightly less packet delivery ratio as compared to AOMDV. Second, NDj-AODV suffers from larger end-to-end delays than AODV but lesser than AOMDV. Third, for higher mobility values, the NDj-AODV incurs high delays due to selecting various routes to a destination in case of link breakages.

\section{J. QoS Routing Protocol with Multiple Node-Disjoint Paths in Ad Hoc Networks}

$\mathrm{Su}$ and Tzeng [31] proposed node-disjoint multi-path quality-of-service (QoS) AODV protocol based on AODV and SPAC routing protocols with slight modifications in the RREQ and Hello packets. This protocol provides efficient bandwidth estimations and admission control. The multi-path QoS AODV protocol uses back-up routes to avoid making wasteful route discovery while detecting link failure; in addition, the protocol can combine multiple routes to support QoS transmission if a single route with sufficient bandwidth cannot be discovered.

Once the source node receives a data flow request from its application layer, the first step is to determine the bandwidth requirement (BWQ) of this data flow. Then, the source node begins the bandwidth check and establishes a virtual connection to the destination node. In practice, the interference range is usually greater than the communication range, and the strength of the interference power is inversely proportional to the square of the distance. For example, as in Fig. 6, nodes A, C, and F are in node B's communication range, whereas nodes $\mathrm{A}, \mathrm{C}, \mathrm{D}, \mathrm{F}$, and $\mathrm{S}$ are in node $\mathrm{B}$ 's interference range. Multiple nodes on the route of a flow may contend for bandwidth at one node, and each of these nodes consumes an amount of bandwidth (BWQ) at this node. The 
number of these types of nodes is called the contention count (Nct) of the route. The Nct equalsh $+d$, where $h$ is the number of nodes which are prior to node $\mathrm{B}$ and are in the interference range of node $B$ (i.e. $h$ is the number of hops from the source node) and $d$ is the number of hops from node $B$ to the destination on the route.

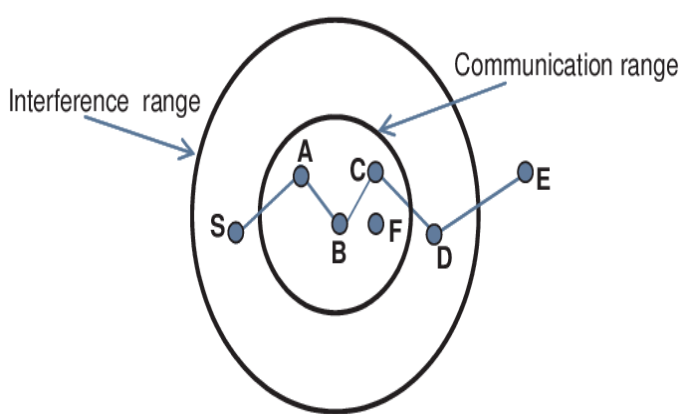

Fig. 6. Limits of node B's communication range and interference range.

When a source node needs to start the communication, it broadcasts an RREQ packet to its neighbors. The RREQ contains the source Id, source sequence, destination Id, hop count, BWQ, Temp_RBWn. The source Id, source sequence is defined as the "Flow Id" used to denote the data flow. Hop count, BWQ, and Temp_RBW $\mathrm{n}$ are the number of hops, the bandwidth requirement of the data flow, and the minimum residual bandwidth between the source node and the intermediate node $\mathrm{n}$, respectively. Temp_RBW $\mathrm{n}$ is defined as:

$$
\begin{aligned}
& \text { Temp_RBW }=\operatorname{Min}\left(\frac{M i n_{\_} R B W_{\text {source }}}{N c t_{\text {source }}}, \frac{M i n_{-} R B W_{2}}{N c t_{2}}, \ldots\right. \\
&\left.\ldots . . \frac{M i n_{-} R B W_{n}}{N c t_{n}}\right)
\end{aligned}
$$

When an intermediate node receives the RREQ, the first step is to check the "flow id" field of the RREQ whether it contains the same flow id. If they are different, the intermediate node re-calculates Nct and Temp_RBW, and then builds up the temp table. Finally, the re-broadcasting temp table records of RREQ containing the flow id, previous node id, hop count, Temp_ RBW, and each temp entry denotes a partial route information. If the same flow id is checked, the intermediate node re-calculates Nct and Temp_RBW. If all Temp_RBWs in the temp table (same flow id) are less than BWQ, and the new Temp_RBW is larger than BWQ, this node will update the temp table and then re-broadcast RREQ; otherwise, it only re-broadcasts RREQ. This procedure results in multiple temp entries for one flow id and many route selections. Each node sends RREQ at most twice to avoid excessive overhead.

When a destination node receives RREQ, it checks whether the "flow id" field of RREQ contains the same flow id. If they are different, the node sets up the timer and accepts the RREQ with the same flow id before the timer expires. After the timer expires, it discards the RREQ. If RREQ is successfully accepted, it re-calculates new Temp_RBW. If the new Temp_RBW is larger than BWQ, thus the single route can satisfy the bandwidth requirement, and then sends RREP back to the source node. If RREQ is with duplicate flow id and satisfies the bandwidth requirement, the destination will send RREP back. If not, store the information of RREQ in the temp table. After the timer expires, if the destination node does not send RREP, then start the proposed method of "Route combination". Route combination means that multiple temp entries are combined together. If the total amount of Temp_RBW is larger than BWQ, thus multiple routes with sufficient bandwidth can be found. If not, do nothing. The temp entry is selected by two conditions: better bandwidth or smaller hop count.

After an intermediate node receives the RREP, it checks the "flow id" field of RREP to verify if it contains the same flow id. If they are different, the node will build a routing table. The routing table entry contains: $<$ flow id, next node id, hop count, Route_RBW>. Afterward, the node chooses a route from the temp routing table and forwards RREP by two ways. First, it chooses a route with better bandwidth; however, if more than one route contains the same value of bandwidth, it compares the route's hop-count to make the decision. Second, it chooses the route with better hop-count; however, if more than one route contains the same value of hop-count, it compares the route's value of bandwidth to make the decision. After deciding a route from the temp entry, the node re-calculates Nct and Route_RBW. Finally, the node sends RREP back to the source and deletes the temp entry.

When the source node receives RREP, the first step is to check the "flow id" field in the RREP. If the flow id is the same and the timer does not expire, the RREP is accepted; if not, the RREPis discarded. Upon receiving the RREP, the source node re-calculates the new Route_RBW. If new Route_RBW is greater than BWQ, thus the source node does find a single route to meet the bandwidth requirement. If not, store the information of RREP in the temp table. Afterward, the source node checks the main table for that flow id. If it already exists, the source node will build up the backup route in the backup table for this flow id. If not, the source node builds up the main table for this flow id. After the timer expires, the source node will check the Route_RBW to be with the same flow id in the temp table, and try to combine those Route_RBWs to meet the bandwidth requirement. If the source node can find multiple Route_RBWs for a route with sufficient bandwidth, the source node will build the main route table for this flow id. If the main route table has this flow id, the source node will build a backup table for this flow id.

The multipath QoS AODV protocol has two drawbacks as follows. First, when the number of flows is 10, the multi-path QoS AODV (BW \& HP), SPAC and AODV produce similar throughput and a small number of blocking flows. Second, the multi-path QoS AODV increases overhead because it uses more control messages to establish multiple paths.

\section{K. Comparison}

AODV is an improvement of the Destination-Sequenced Distance Vector routing (DSDV) [32] protocol. AODV aims to reduce the number of broadcast messages forwarded throughout the network by discovering routes on-demand instead of keeping a complete up-to-date routing table. The advantages of AODV protocol are that it favors the least congested route instead of the shortest route and it supports both unicast and multicast packet transmissions even for 
nodes in constant movement. It also responds very quickly to the topological changes that affect the active routes.

On the other hand, one of the major problems of AODV is the maintenance of only one route per destination. This means that every time a path is broken, AODV has to initiate a route discovery process, which leads to more overhead, higher delays and higher packet loss in the network.

TABLE I: Multipath Routing PROTOCOLS ExTENSION OF AODV

\begin{tabular}{|c|c|c|c|c|}
\hline Protocols & Tools & Objective & $\begin{array}{c}\text { Num. } \\
\text { of } \\
\text { Paths }\end{array}$ & Drawback \\
\hline $\begin{array}{l}\text { AOMDV } \\
(2001)\end{array}$ & $\begin{array}{l}\text { Use multiple loop free } \\
\text { (advertised hop count) } \\
\text { and link-disjoint paths. }\end{array}$ & $\begin{array}{l}\text { Provide efficient fault tolerance in } \\
\text { the sense of fast and efficient } \\
\text { recovery from route failures in } \\
\text { dynamic networks. }\end{array}$ & 3 & $\begin{array}{l}\text { When increasing the network density, the protocol } \\
\text { performance decreases and it has the additional } \\
\text { overhead of more RREPs per route discovery. }\end{array}$ \\
\hline $\begin{array}{l}\text { AODVM } \\
(2003)\end{array}$ & $\begin{array}{l}\text { Use reliable nodes and } \\
\text { last hop ID. }\end{array}$ & $\begin{array}{l}\text { Design a multipath routing } \\
\text { framework for providing enhanced } \\
\text { robustness to node failures. }\end{array}$ & $\begin{array}{l}\text { At least } 3 \\
\text { or } 4\end{array}$ & $\begin{array}{l}\text { 1. A sufficient number of node disjoint paths cannot be } \\
\text { found without incurring a large amount of overhead. } \\
\text { 2. It can only achieve better performance in scenarios } \\
\text { with lower mobility and higher node density. } \\
\text { 3. It severely suffers from packet loss when the } \\
\text { network becomes dynamic. }\end{array}$ \\
\hline $\begin{array}{l}\text { AODVM/PD } \\
\quad(2005)\end{array}$ & $\begin{array}{l}\text { Depend on correlation } \\
\text { factors(LCF, ACF and } \\
\text { CT). }\end{array}$ & $\begin{array}{l}\text { Provide better fault tolerance and } \\
\text { smaller end-to-end delay than } \\
\text { AODVM in networks with low } \\
\text { mobility. }\end{array}$ & 3 & $\begin{array}{l}\text { 1. With more nodes moving, the delay becomes greater } \\
\text { than AODVM. } \\
\text { 2. The control overhead is increased due to the } \\
\text { transmission of CORR packets during the route } \\
\text { discovery phase. }\end{array}$ \\
\hline $\begin{array}{l}\text { AODV-ABR } \\
\quad(2007)\end{array}$ & $\begin{array}{l}\text { Depend } \\
\text { onAODV-ABR and } \\
\text { AODV-ABL. }\end{array}$ & Reduce control overhead. & $\begin{array}{l}\text { Backup } \\
\text { route }\end{array}$ & $\begin{array}{l}\text { The traffic load becomes heavier when more nodes } \\
\text { participate in a wireless network. The probability of } \\
\text { packet collisions will increase, resulting in the } \\
\text { degradation of overall performance. }\end{array}$ \\
\hline $\begin{array}{l}\text { AM-AOMDV } \\
\qquad(2010)\end{array}$ & $\begin{array}{l}\text { Based on multi-metric } \\
\text { (node-to-end RSSI, } \\
\text { node-to-end latency } \\
\text { and node occupancy) } \\
\text { and local route update. }\end{array}$ & $\begin{array}{l}\text { Increases the packet throughput } \\
\text { and route longevity, decrease the } \\
\text { end-to-end latency, routediscovery } \\
\text { frequency and route overhead } \\
\text { under high mobility environments. }\end{array}$ & $2-5$ & $\begin{array}{l}\text { In a sparse network (i.e., lower number of } \\
\text { connections), the AM-AOMDV has a higher route } \\
\text { discovery delay than AOMDV. }\end{array}$ \\
\hline $\begin{array}{l}\text { CBRD } \\
(2013)\end{array}$ & Depend on queue size. & $\begin{array}{l}\text { Check congestion on a node and } \\
\text { then apply load balancing. }\end{array}$ & $\begin{array}{l}\text { Multiple } \\
\text { link } \\
\text { disjoint } \\
\text { Paths } \\
\end{array}$ & $\begin{array}{l}\text { 1. When a node moves to other side, the delay begins } \\
\text { to increase. } \\
\text { 2. Some packets are lost when a node moves in another } \\
\text { direction. }\end{array}$ \\
\hline $\begin{array}{l}\text { ISPDA } \\
(2013)\end{array}$ & $\begin{array}{l}\text { Depend on node } \\
\text { stability and hop count. }\end{array}$ & $\begin{array}{l}\text { Improve the performance of } \\
\text { SPDA protocol }\end{array}$ & $\begin{array}{c}\text { Ten } \\
\text { partially } \\
\text { disjoint } \\
\text { paths } \\
\end{array}$ & $\begin{array}{l}\text { The discovery overhead of ISPDA is higher than that } \\
\text { of SPDA. }\end{array}$ \\
\hline $\begin{array}{l}\text { MM- AODV } \\
\quad(2014)\end{array}$ & $\begin{array}{l}\text { Depend on neighbors } \\
\text { of source node } \\
\text { (secondary sources) } \\
\text { and neighbors of } \\
\text { destination node. }\end{array}$ & $\begin{array}{l}\text { Discover maximum available } \\
\text { complete node-disjoint paths, } \\
\text { balances energy and traffic load. }\end{array}$ & $\begin{array}{l}\leq \mathrm{S} \text { or } \mathrm{D} \\
\text { neighbors. }\end{array}$ & $\begin{array}{l}\text { 1. When node speed increases, the path break } \\
\text { probability also increases, therefore the packet } \\
\text { delivery rate of MM-AODV become slightly less than } \\
\text { AODV. } \\
\text { 2. The memory storage is increased as the alternative } \\
\text { paths are stored. }\end{array}$ \\
\hline $\begin{array}{l}\text { NDj- AODV } \\
\text { (2014) }\end{array}$ & $\begin{array}{l}\text { Use the concept of } \\
\text { overhearing in wireless } \\
\text { medium. }\end{array}$ & $\begin{array}{l}\text { Find multiple node-disjoint } \\
\text { paths to destination with less } \\
\text { routing overhead and low route } \\
\text { discovery latencies. }\end{array}$ & $\begin{array}{l}\text { Set of } \\
\text { node-disjo } \\
\text { int paths. }\end{array}$ & $\begin{array}{l}\text { 1. It achieves a slightly less packet delivery ratio as } \\
\text { compared to AOMDV. } \\
\text { 2. The NDj-AODV suffers from larger end-to-end } \\
\text { delays as compared to AODV but lesser than } \\
\text { AOMDV. } \\
\text { 3. For higher mobility values, the NDj-AODV incurs } \\
\text { high delays due to selecting various routes to } \\
\text { destination in case of link breakages. }\end{array}$ \\
\hline $\begin{array}{l}\text { Multi-path } \\
\text { QoS AODV } \\
\quad(2015)\end{array}$ & $\begin{array}{l}\text { Depend on } \\
\text { Temp_RBWn, Nct and } \\
\text { BWQ. }\end{array}$ & $\begin{array}{l}\text { Design efficient } \\
\text { bandwidth estimations, admission } \\
\text { control and node- } \\
\text { disjoint multi-path } \\
\text { establishment. }\end{array}$ & $\begin{array}{l}\text { Back-up } \\
\text { routes. }\end{array}$ & $\begin{array}{l}\text { 1. When the number of flows is 10, the multi-path QoS } \\
\text { AODV, SPAC and AODV produces similar } \\
\text { throughput and small number of blocking flows. } \\
\text { 2. The protocol increases overhead because it uses } \\
\text { more control messages to establish multiple paths. }\end{array}$ \\
\hline
\end{tabular}

Tools: mechanism of selecting multiple routes in the protocol.

In order to alleviate the aforementioned problems, a large number of multipath routing protocols were proposed to improve the performance of the AODV protocol under various operating scenarios. Some of these scenarios involve QoS improvement, load balancing, congestion alleviation, fault tolerance and energy balancing.

The main improvement direction has been about the finding of multiple disjoint routes. There are various approaches taken by the protocols, e.g., AOMDV [20] (using advertised hop count), AODVM [9] (based on reliable nodes and last hop ID), AODVM/PD [24] (depending on correlation factors metric), AM-AOMDV [26] (depending on local route update and multi-metric i.e., node-to-end latency, node-to-end RSSI, and node occupancy), CBRD [27] (depending on the queue size of node), MM-AODV [29] (depending on neighbors of source node (secondary sources) and neighbors of destination node), NDj- AODV [30] (using the concept of overhearing in wireless medium), Multi-path QoS AODV [31] (depending on Temp RBWn, Nct and BWQ).

There are other improvement directions such as backup route in AODV-ABR [25] (using AODV-ABR and AODV-ABL), partially disjoint path (ISPDA [28]) (more stable than the maximally disjoint). Table I summarizes the 
protocols reviewed in this section and compares some of their key features.

\section{CONCLUSIONS AND Future WORK}

Multipath routing has been a promising technique in MANETs. As opposed to their single path (e.g. AODV) counterparts, on-demand routing protocols with multipath capability can effectively deal with mobility-induced link failures in mobile ad hoc networks. The outcome of this fact is the multipath routing protocols that have been proposed for mobile ad hoc networks throughout the decades. The technique proposes that the traffic can be distributed and carried by multiple simultaneously available paths so that the available bandwidth can be better utilized by using multiple active transmission tasks, especially under low traffic load conditions. It also provides a better fault tolerance for the system if a path fails.

In this survey, we have reviewed and compared around ten representative protocols. While different kinds of protocols operate under different scenarios, they usually share the common goal to reduce control packet overhead, maximize throughput, and minimize the end-to-end delay. The main differentiating factor between the protocols is the ways of finding and maintaining the routes between source-destination pairs.

Table I provides a summary of all the multipath routing protocols reviewed in this paper and provides a checklist that can help network administrators to choose a suitable multipath routing protocol meeting more than one performance objective.

To the best of our knowledge, we have grouped and summarized the weaknesses of the multipath routing protocols as mentioned in Table I. These typical weaknesses include the increased end-to-end delay, high processing complexity, large number of overhead packets, the increased power consumption, small data rate per route, the decreased performance when the network density increases, high packet loss, the increased packet size, the increased packet collisions and performance degradation, the reduced packet delivery ratio and throughput when the network size increases.

Our future work will focus on the design of multipath routing protocols that will overcome the aforementioned weaknesses listed in Table I.

\section{ACKNOWLEDGMENT}

This work is supported by the National Science Foundation for Distinguished Young Scholars of China under Grant No. 61225012 and No. 71325002.

\section{REFERENCES}

[1] N. Z. Ali, R. B. Ahmad, and S. A. Aljunid, "A survey on on-deman multipath routing protocol in MANETs," in Proc. International Conference on Electronic Design, IEEE, 2008.

[2] S. K. Sarkar, T. G. Basavaraju, and C. Puttamadappa, "Ad hoc mobile wireless networks principles, protocols, and applications," USA, Auerbach publications, 2013, ch. 1, pp. 21-23.

[3] S. Mueller, R. P. Tsang, and D. Ghosal, Multipath Routing in Mobile Ad Hoc Networks: Issues and Challenges, Springer-Verlag, Berlin Heidelberg, pp. 209-234, 2004.

[4] B. Soujanya, T. Sitamahalakshmi, and C. D. AKAR, "Study of Routing Protocols in Mobile Ad-Hoc Networks," International Journal of
Engineering Science and Technology (IJEST), vol. 3, no. 4, pp. 2622-2631, 2011.

[5] D. B. Johnson, D. A. Maltz, and J. Broch, "DSR: The dynamic source routing protocol for multi-hop wireless ad hoc networks," in C. E. Perkins Ed., Ad Hoc Networking, Addison-Wesley, pp. 139-172, 2001.

[6] C. E. Perkins and E. M. Royer, "Ad hoc on-demand distance vector routing," in: Proc. the Second IEEE Workshop on Mobile Computing Systems and Applications, pp. 90-100, 1999.

[7] A. Hassan, "Simulations on multipath routing based on source routing," Bachelor thesis, Bern University, 2008.

[8] T. Okazaki, E. Kohno, T. Ohta, and Y. Kakuda, "A multipath routing method with dynamic ID for reduction of routing load in ad hoc networks," Institute for Computer Sciences, Social Informatics and Telecommunications Engineering, pp. 114-129, 2010

[9] Z. Ye, S. V. Krishnamurthy, and S. K. Tripathi, "A framework for reliable routing in mobile ad hoc networks," IEEE INFOCOM, pp. 270-280, 2003.

[10] M. Tarique, K. Tepe, S. Adibi, and S. Erfani, "Survey of multipath routing protocols for mobile ad hoc networks," Journal of Network and Computer Applications, pp. 1125-1143, 2009.

[11] N. A. Husieen, "A reliable multipath dynamic source routing protocol for multimedia applications in mobile ad-hoc networks," Doctor of Philosophy, University Utara Malaysia, 2013.

[12] R. Kaur, R. Mahajan, and A. Singh, "A survey on multipath routing protocols for MANETS," IJETTCS, vol. 2, issue 2, pp. 42-45, 2013.

[13] S.-J. Lee and M. Gerla, "Split multipath routing with maximally disjoint paths in ad hoc networks," IEEE, pp. 3201-3205, 2001.

[14] W. Lou, W. Liu, and Y. Zhang, "Performance optimization using multipath routing in mobile ad hoc and wireless sensor networks," Kluwer Academic, pp. 1-29, 2005.

[15] V. B. Kute and M. U. Kharat, "Survey on QoS for multi-path routing protocols in mobile ad-hoc networks," International Journal of Machine Learning and Computing, vol. 2, no. 4, pp. 458-461, 2012.

[16] H. Gharavi, "Multichannel mobile ad hoc links for multimedia communications," IEEE, vol. 96, no. 1, pp. 77-95, 2008.

[17] Xin Wang, "Mobile ad-hoc networks: Protocol design," India, InTech, 2011, ch. 17, pp. 327-328

[18] A. A. Etorban, "The design and performance evaluation of a proactive multipath routing protocol for mobile ad hoc networks," Ph.D. dissertation, Heriot-Watt University, 2012.

[19] A. Boukerche, B. Turgut, N. Aydin, and M. Z. Ahmad, "Routing protocols in ad hoc networks: A survey," Computer Networks, pp. 3032-3080, 2011.

[20] M. K. Marina and S. R. Das, "On-demand multipath distance vector routing in ad hoc networks," IEEE, pp. 14-23, 2001.

[21] B. Kan and J. Fan, "Interference activity aware multi-path routing protocol," EURASIP Journal on Wireless Communication and Network, pp. 1-13, 2012.

[22] G. Parissidis, V. Lenders, M. May, and B. Plattner, "Multi-path routing protocols in wireless mobile ad hoc networks: A quantitative comparison," Springer Berlin Heidelberg, pp. 313-326, 2006.

[23] N. Nithya and R. Maruthaveni, "A survey on routing protocols in mobile ad hoc networks," IJSR, vol. 2, issue 1, pp. 350-354, 2013.

[24] S. Mueller and D. Ghosal, "Analysis of a distributed algorithm to determine multiple routes with path diversity in ad hoc networks," IEEE, pp. 277-285, 2005.

[25] W. K. Lai, S.-Yu Hsiao, and Y.-Chung Lin, "Adaptive backup routing for ad-hoc networks," Computer Communications, pp. 453-464, 2007.

[26] S. Khimsara, K. K. R. Kambhatla, J. Hwang, S. Kumar, and J. D. Matyjas, "AM-AOMDV: Adaptive multi-metric ad-hoc on-demand multipath distance vector routing," ICST Institute for Computer Sciences, Social-informatics and Telecommunications Engineering, pp. 884-895, 2010.

[27] O. S. Bawa and S. Banerjee, "Congestion based route discovery AOMDV protocol," International Journal of Computer Trends and Technology, vol. 4, no. 1, pp. 54-58, 2013.

[28] W. Almobaideen, D. Al-Khateeb, A. Sleit, M. Qatawneh, K. Qadadeh, R. Al-Khdour, and H. A.Hafeeza, "Improved stability based partially disjoint AOMDV," Int. J. Communications, Network and System Sciences, pp. 244-250, 2013.

[29] T. Chowdhury and R. B. M. Mukta, "A novel approach to find the complete node-disjoint multipath in AODV," presented at 3rd International Conference on Informatics, Electronics \& Vision, IEEE, 2014.

[30] V. Arya and C. Gandhi, "NDj - AODV: Node disjoint multipath routing in mobile ad hoc networks based on AODV protocol," IEEE, 2014.

[31] Y.-W. Su and S.-S. Tzeng, "QoS routing protocol with multiple node-disjoint paths in ad hoc networks," Wireless Pers Commun, Springer, vol. 83, no. 3, pp. 1867-1885, 2015. 
[32] C. E. Perkins and P. Bhagwat, "Highly dynamic destination-sequenced distance-vector routing (DSDV) for mobile computers," ACM SIGCOMM, August-September, pp. 234-244, 1994.

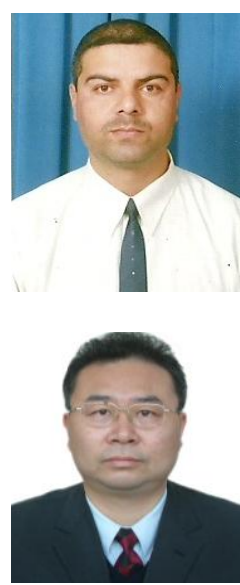

Tariq A. Murshedi received the BSc and the MSc degrees from the Department of Computer Science at Mustansiriyah University, Baghdad, Iraq, in 1992, and 2006. He is a Ph.D student in the Department of Computer Science at Northeastern University, Shenyang, China. He is also an instructor in Cisco Networking Academy, Babylon University, Iraq. His research interests include routing protocols in Mobile ad hoc networks and wireless network.

Xingwei Wang received the Ph.D. degree in computer science from Northeastern University, Shenyang, China, in 1998. He is currently a professor in the Faculty of Information Science and Engineering, Northeastern University. His research interests include optimized routing algorithms in new generation internet and future internet, resource management for cloud computing.

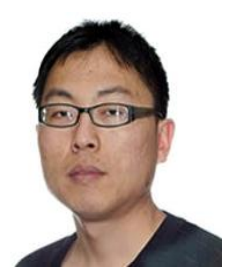

Hui Cheng received the BSc and the MSc degrees in computer science from Northeastern University, China in 2001 and 2004, and the PhD degree in computer science from the Hong Kong Polytechnic University, Hong Kong in 2007. From January 2008 to July 2010, he was employed as a research associate at University of Leicester, UK. He joined the Department of Computer Science and Technology at University of Bedfordshire as a lecturer in October2010. He joined School of Computing \& Mathematical Sciences at Liverpool John Moores University as a senior lecturer in October 2013. His research interests include artificial intelligence, dynamic optimization, optical networks, mobile ad hoc networks, QoS routing. 